\title{
NMR relaxation measurements of biofouling in model and geological porous media
}

\author{
Sarah L. Codd ${ }^{\mathrm{a}, \mathrm{c}, *}$, Sarah J. Vogt ${ }^{\mathrm{b}, \mathrm{c}}$, Jennifer A. Hornemann ${ }^{\mathrm{b}, \mathrm{c}}$, Adrienne J. Phillips ${ }^{\mathrm{c}}$, James E. Maneval ${ }^{\mathrm{d}}$, \\ Konstantin R. Romanenko a,b, Logan Hansen ${ }^{\mathrm{e}}$, Alfred B. Cunningham ${ }^{\mathrm{c}}$, Joseph D. Seymour ${ }^{\mathrm{b}, \mathrm{c}}$
}

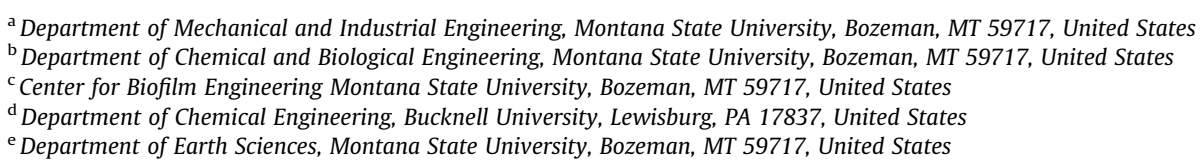

\section{A R T I C L E I N F O}

\section{Article history:}

Received 19 November 2010

Received in revised form 1 February 2011

Accepted 14 March 2011

Available online 21 March 2011

\begin{abstract}
A B S T R A C T
Recently 2D nuclear magnetic resonance (NMR) relaxation techniques have been able to access changes in pore structures through surface and diffusion based relaxation measurements. This research investigates the applicability of these methods for measuring pore and surface changes due to biofilm growth in various model porous systems and natural geological media. Model bead packs of various construction containing $100 \mu \mathrm{m}$ borosilicate and soda lime glass beads were used to demonstrate how changes in the measured relaxation rates can be used to non-invasively verify and quantify biofilm growth in porous media. However significant challenges are shown to arise when trying to implement the same techniques to verify biofilm growth in a natural geological media.
\end{abstract}

(c) 2011 Elsevier Ltd. All rights reserved.

\section{Introduction}

From water service utilities to food processing, biofilms either create havoc or are crucial to system function in almost every water based industrial process. Bio-barriers, a traditional method in which biofilm establishment and maintenance is crucial, are used around the world to improve the quality of our environment by degrading or containing chemical contaminants (Cunningham et al., 2003). Additionally biofilms are now being investigated to serve as buffers to sequester $\mathrm{CO}_{2}$ in an effort to curb the effects of global warming (Mitchell et al., 2008b), requiring that biofilms grow and survive intact in naturally occurring porous geological structures. Hydrodynamics and mass transport inside biofouled porous media have long been of interest due to their broad importance in nature, industry and medicine. Since the early 1990s, researchers have used a variety of porous systems, e.g. sand, glass bead packs and flat plate channel reactors, to measure how the extracellular polymeric substance (EPS) production from bacterial cells changes a system's hydraulic conductivity, mass transport and dynamic flow patterns (Seymour et al., 2004; Sharp et al., 2005). Using NMR to study how biofouling affects transport in porous media is a relatively new application. NMR imaging has been used to spatially resolve biofouled porous systems (Hoskins et al.,

\footnotetext{
* Corresponding author at: Department of Mechanical and Industrial Engineering Montana State University, MT 59717-3800, United States. Tel.: +1 4069941944.

E-mail address: scodd@coe.montana.edu (S.L. Codd).
}

1999; Paterson-Beedle et al., 2001), and monitor flow changes in biofouled bead packs (Seymour et al., 2004; von der Schulenburg et al., 2008).

To better understand the structure and transport changes that occur in biofouled porous media, this study investigates the use of $1 \mathrm{D}$ and 2D nuclear magnetic resonance (NMR) relaxation measurement methods (Fleury and Soualem, 2009; Godefroy et al., 2001; Jaeger et al., 2010; Mitchell et al., 2007; Monteilhet et al., 2006; Song et al., 2002, 2008; Washburn and Callaghan, 2006) to observe changes that verify biofilm growth and assess the extent and distribution of this growth. The impact of nutrient growth media chemistry on NMR relaxation measurements were observed and quantified in the porous systems, both model bead packs and natural Berea sandstone. Similar model glass bead systems have previously been used to quantify water exchange between different pore surfaces (Mitchell et al., 2007) and similar rock samples have been used to quantify exchange between different pores (Washburn and Callaghan, 2006). A recent study using a low-field NMR system of both glass bead systems and a soil reactor found a $T_{2}$ dependence on biofilm growth (Bayer et al., 2010). In pursuit of developing an NMR biosensor for porous geological structures, the specific objectives of this study were to show the richness of the NMR $T_{2}-T_{2}$ and $T_{1}-T_{2}$ distributions and in particular investigate whether changes in $T_{2}$ and $T_{1}$ distributions and the presence, absence or change of the off diagonal, or exchange, peaks as a function of exchange time can detect the presence of biofilm, spatial extent of biofouling and ultimately determine its impact on the transport properties inside the porous media. 


\section{Theory}

\section{1. $T_{2}$ relaxation}

The $T_{2}$ for a liquid filled pore is influenced by the diffusion of the liquid molecules, presence of relaxation sinks (bound water, paramagnetic impurities, biofilm macromolecules) and field inhomogeneities due to susceptibility differences and paramagnetic impurities (Brownstein and Tarr, 1979; Godefroy et al., 2001). If field inhomogeneities can be neglected, Brownstein and Tarr (1979) have shown that the $T_{2}$ relaxation distribution observed in porous media can be understood by solving an eigenvalue problem for water diffusing with molecular diffusion $D$ in pores of size $a$ and with surface relaxation $\rho_{2}$. The measured $T_{2}$ relaxation distribution is dominated by the longest $T_{2}$ modes and depends on the ratio of $\rho_{2} a / D$.

If the relaxation is surface limited, the fast exchange limit (Brownstein and Tarr, 1979) is such that $\rho_{2} a / D \ll 1$ and only one relaxation eigenmode dominates and the relaxation can be shown to be proportional to the surface to volume ratio $S / V$ or pore size $a=V / S$. At the other limit, if the relaxation is diffusion limited, the slow exchange limit (Brownstein and Tarr, 1979) is such that $\rho_{2} a / D \gg 10$, then the relaxation can be shown to be proportional to both the diffusion coefficient and the square of the pore size (Brownstein and Tarr, 1979) and several of the first few eigenmodes can contribute significantly leading to the possibility of multi-exponential signal decay even in a single pore.

Multi-exponential signal decay can also be observed due to the presence of macromolecules and is dependent on the timescale of the proton-proton exchange between the macromolecules and the water. An additional cause of multi-exponential decay can be spatially varying and strong field gradients due to magnetic field susceptibilities induced by the difference in magnetic susceptibility of the pore liquid and porous media solid matrix.

If multiple modes of relaxation exist, then in addition to the observation of multi-exponential signal decay there can be multiple coupled $T_{2}$ populations. At the two extremes, these populations are weakly coupled if exchange between the populations cannot take place during the lifetime of the excited NMR signal, or strongly coupled if the exchange is so rapid that only one average $T_{2}$ is measurable (Brownstein and Tarr, 1979; Washburn and Callaghan, 2006; Fleury and Soualem, 2009).

The timescale of the transition between the slow and the fast exchange regimes depends on the time necessary for the spins to diffusively sample the entire pore region and/or all relaxation sites. Hence, the measured $T_{2}$ distribution for a particular timescale depends on the pore surface, presence of macromolecules, the pore $S / V$ and the viscosity of the imbibed fluid.

\section{2. $T_{2}-T_{2}$ exchange experiments}

2D NMR $T_{2}-T_{2}$ distributions have the ability to probe exchange between different $T_{2}$ regions if the ratio of $T_{1} / T_{2}>1$ and if the exchange time between the two encoding periods is appropriately chosen (Monteilhet et al., 2006; Song et al., 2008). If present, the off diagonal, or exchange, peaks can yield information related to the spins diffusive transport between several different $T_{2}$ regions and these different $T_{2}$ regions may represent different size pores (Washburn and Callaghan, 2006) or different surfaces (Mitchell et al., 2007), although they can also represent the strongest eigenmodes of the relaxation in a single pore (Song et al., 2008). Biofilm growth in a porous media complicates the system by adding a gel phase, the extracellular polymeric substance (EPS), to the pore walls and biomacromolecules to the fluid thus changing pore connectivity, effective pore size, pore surface chemistry and bulk $T_{2}$, as well as introducing salts, macromolecules and paramagnetic impurities via the bacterial growth medium. In order to interpret $T_{2}-T_{2}$ measurements, a conceptual physical understanding of the relaxation taking place within the pore spaces is needed. As indicated above, relaxation is a function of the viscosity of the fluid, proton exchange between molecular sites, presence of macromolecules, pore size, the physical and chemical nature of the pore walls (bead surfaces) and any magnetic field inhomogeneities caused by magnetic susceptibility. Thus, changing system properties, such as adding salts and macromolecules present in microbial growth media to the bulk fluid or biofouling the bead pack, alters relaxation mechanisms. Other impacts of biofouling on NMR relaxation include changes in water-polymer proton exchange due to the immobility of polymers cross linked in the EPS matrix. A water molecule's movement in a heterogeneous system alters the magnetic and chemical environment it experiences and protons on the water molecule engage in rapid exchange with slowly moving semi-rigid protons on macromolecules (Vaca Chavez et al., 2006). This generates a measurable effect on both $T_{1}$ and $T_{2}$.

The NMR pulse sequence for the $T_{2}-T_{2}$ exchange experiment (Lee et al., 1993) is shown in Fig. 1. The initial $90^{\circ} \mathrm{rf}$ pulse excites the system and tips the macroscopic magnetization into the transverse plane and is then refocused using a CPMG sequence of $180^{\circ} \mathrm{rf}$ pulses to minimize susceptibility gradient effects and other inhomogeneous broadening effects. Relaxation due to spin-lattice $T_{1}$ and spin-spin $T_{2}$ interactions will occur during this time. The magnetization is then stored along the longitudinal axis for an exchange period $\tau_{m}$ during which only $T_{1}$ relaxation impacts the magnetization. Displacement of the water molecules can occur over this exchange time period which is on the scale of $1 \mathrm{~ms}$ to $1 \mathrm{~s}$. After the exchange time period the magnetization is returned to the transverse plane and a second CPMG sequence of $180^{\circ} \mathrm{rf}$ pulses is applied and data is acquired for each echo. The number of pulses in the second CPMG sequence (" $n$ " in Fig. 1) is the same throughout the experiment, while the number of pulses in the CPMG before the exchange period (" $m$ " in Fig. 1) is changed logarithmically. A 16 step phase cycle was used to ensure that only molecules that experience all of the rf pulses will contribute to the final data. The result is that the $T_{2}-T_{2}$ sequence encodes for the $T_{2}$ distribution before and after the exchange time period (Lee et al., 1993). Inverse Laplace transformation (ILT) of the data produces 2D relaxation maps where collapse of the data onto each axis also provides a $1 \mathrm{D} T_{2}$ relaxation time distribution of the system. Peaks along the diagonal represent water molecules that have remained in a similar $T_{2}$ relaxation time environment during the exchange time and off diagonal peaks represent water molecules that have diffused to a different $T_{2}$ relaxation time environment. The data processing using the inverse Laplace transformation was executed using the same parameters for all compared data sets, including the step sizes, smoothing parameter and time range.

Several models have been proposed to provide interpretation of off diagonal, or exchange, peaks observed in porous media that grow as a function of the exchange time, see Fig 2 . The pore to pore exchange model (Washburn and Callaghan, 2006) considers two pores of different sizes. More collisions with the pore's surface occur for water molecules in smaller pores (due to the higher $S / V$ ratio) and this enhances relaxation giving a shorter $T_{2}$ relaxation time. Thus, pore size distribution can be determined from the $T_{2}$ distribution, due to the $T_{2}$ dependence on $S / V \sim 1 / a$ (Brownstein and Tarr, 1979). In a homogeneous bead pack where pores are of relatively the same size, the smaller pore size can be the throat connecting the pores (Washburn and Callaghan, 2006).

The single pore model (Song et al., 2008), differs slightly and is based on the eigenmode expansion of the relaxation, as recently demonstrated using a model in which a single pore size generated exchange peaks in $T_{2}-T_{2}$ simulations when the $T_{1} / T_{2}$ ratio was large 


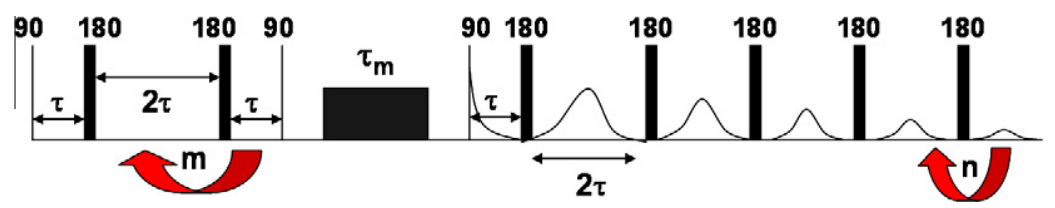

Fig. 1. $T_{2}-T_{2}$ experiment pulse sequence.
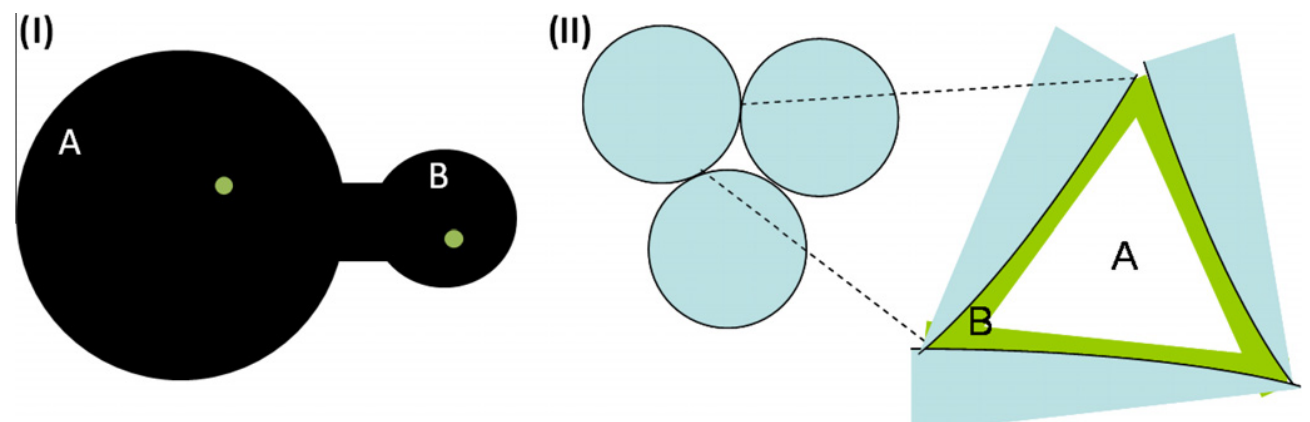

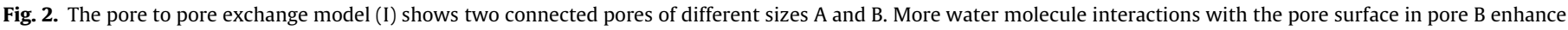

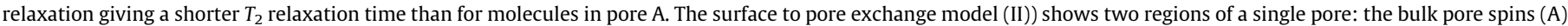

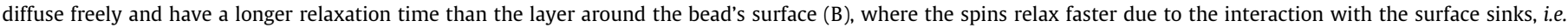
bound water or macromolecules.

enough (Song et al., 2008). Another model considers that two distinct $T_{2}$ populations come from the bulk pore spins, where they diffuse freely close to that of unrestricted water, and a layer around the bead's surface, where the spins relax much faster due to the interaction with the surface sinks or due to a region of strong susceptibility induced field gradients near pore boundaries.

Regardless of the model used, as long as the two $T_{2}$ populations are not so strongly coupled that only one value is measurable, the hypothetical results shown in Fig. 3 for a short and a long exchange time apply. For short $\tau_{m}$ the slow (A) and fast (B) relaxing populations show up distinctly along the diagonal because there has been very little diffusive motion. However, for longer $\tau_{m}$, the liquid molecules have enough time to sample both the slow and fast relaxation regions and off diagonal, or exchange, peaks appear. These exchange peaks represent water molecules that have moved from one $T_{2}$ domain to another during the exchange time. By measuring the growth rate of the exchange peaks as a function of the exchange time, $\tau_{m}$, it is possible to determine information about the pore structure and connectivity (Washburn and Callaghan, 2006).

The presence of exchange peaks means the two corresponding $T_{2}$ regions are coupled (Fleury and Soualem, 2009). However, an additional criterion for observation of off diagonal peaks is that $T_{1} / T_{2} \gg 1$ for at least one of the regions (Song et al., 2008). In
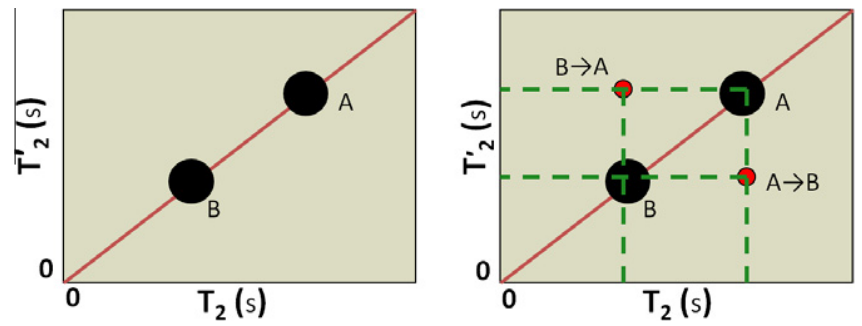

Fig. 3. For a sample with two distinct $T_{2}$ relaxation time populations, for a short exchange time $\left(\tau_{m}\right)$ all the signal appears along the diagonal (left) with two peaks corresponding to the slow (A) and fast (B) relaxation times. For a long exchange time $\left(\tau_{m}\right)$ off diagonal exchange peaks appear representing molecules that have moved from one $T_{2}$ region to the other in the time $\tau_{m}$. systems that have multiple relaxation regions, the lack of off diagonal, or exchange, peaks can mean one of three situations exist: (1) the coupling between the regions is weak or zero over the observed exchange time resulting in no transfer of molecules between populations, (2) the coupling between the regions is very strong and only one average $T_{2}$ value is measureable or (3) $T_{1} / T_{2}$ is too small and the exchange peaks are not measurable (Fleury and Soualem, 2009).

\section{Materials and methods}

\subsection{Glass bead packs}

Three model porous systems were created from $\sim 100 \mu \mathrm{m}$ glass beads (Q.A. Equipment, Ltd., UK). The first bead pack contained borosilicate (BS) glass beads, the second contained soda lime (SL) glass beads and the third contained a randomised mixture of both types ( $\mathrm{BS}+\mathrm{SL}$ ). The $\mathrm{BS}$ glass beads are composed of $70 \% \mathrm{SiO}_{2}, 10 \%$ $\mathrm{B}_{2} \mathrm{O}_{3}, 8 \% \mathrm{Na}_{2} \mathrm{O}, 8 \% \mathrm{~K}_{2} \mathrm{O}, 1 \% \mathrm{CaO}$ and have relatively smooth surfaces. The SL glass beads are composed of $73 \% \mathrm{SiO}_{2}, 13.5 \% \mathrm{Na}_{2} \mathrm{O}$, $0.3 \% \mathrm{~K}_{2} \mathrm{O}, 10.7 \% \mathrm{CaO}, 1.8 \% \mathrm{Al}_{2} \mathrm{O}_{3}$, and $0.4 \% \mathrm{MgO}$. Due to the composition the SL surface has increased surface relaxation evidenced by the difference in both the measured $T_{1}$ and $T_{2}$ relaxation values. Water in the BS bead pack had $T_{1}=2.02 \mathrm{~s}, T_{2}=0.6 \mathrm{~s}$, and $T_{1} /$ $T_{2} \sim 3$. Water in the soda lime (SL) bead pack had a $T_{1}=0.87 \mathrm{~s}$ and a $T_{2}=0.1 \mathrm{~s}, T_{1} / T_{2} \sim 9$. Thus, water relaxes much faster when in contact with SL glass beads as compared to BS glass beads of the same size. The bead packs were analysed using NMR whilst saturated with pure water, then with the Brain Heart Infusion (BHI) growth media, a broth containing proteins, carbohydrates and salt, and finally after inoculation and biofouling. For consistency, the water and BHI saturated systems contained the exact same beads and bead packing, while the biofouled bead packs required repacking of the same beads in a flow capillary.

\subsection{Biofouled glass bead packs}

The biofouled bead pack contained a Bacillus mojavensis biofilm grown under flow conditions in the BHI growth media. 
B. mojavensis is a bacteria of choice for potential $\mathrm{CO}_{2}$ sequestration, because it is naturally occurring in Berea sandstone, forms thick biofilms with a plethora of EPS and is resilient to super critical $\mathrm{CO}_{2}$ (Mitchell et al., 2008a,b). The stages of growing a B. mojavensis biofilm include growth of suspended bacterial cells, inoculation of a bioreactor system and a monitored growth period in BHI growth media. Combining $100 \mathrm{ml}$ of the sterile BHI growth media with $1.0 \mathrm{ml}$ of frozen stock $B$. mojavensis cells, the suspended bacteria cells were shaken at $150 \mathrm{rpm}$ and incubated at $30^{\circ} \mathrm{C}$ for $12 \mathrm{~h}$. The bioreactor, in these experiments either a bead pack or a Berea rock core, was fed via a sterile HPLC pump (Acuflow Series III) with $\mathrm{BHI}$ growth media at $1 \mathrm{ml} / \mathrm{h}$ for $4 \mathrm{~h}$ to ensure saturation of the bead pack before inoculation of suspended $B$. mojavensis culture immediately upstream of the bioreactor. The bacteria were given $4 \mathrm{~h}$ to attach to the surface without flow and then the biofilm was grown for 24-48 $\mathrm{h}$ under flow conditions.

\subsection{Rock core sample}

Similar experiments were performed on a natural Berea sandstone porous media. Samples were $6 \mathrm{~mm}$ in diameter and ranged from $10-20 \mathrm{~mm}$ in length. The rocks were saturated under pressure driven flow with either brine $(40 \mathrm{~g} / 1 \mathrm{NaCl}$ in distilled water) or BHI growth media or were biofouled with B. mojavensis for 24-36 h. The rocks were then removed from the flow loop, wrapped in Teflon tape and tested in a $10 \mathrm{~mm}$ NMR tube.

\subsection{NMR experiments}

NMR measurements were made using a Bruker AVANCE $300 \mathrm{MHz}$ spectrometer, a $5 \mathrm{~mm}$ saddle radio frequency (rf) coil and available magnetic field gradient of $1.7 \mathrm{~T} / \mathrm{m} . T_{2}-T_{2}$ relaxation measurements were acquired using the Altered Relaxation Times allow Detection of Exchange Correlation (ARTDECO) sequence (Lee et al., 1993), more recently referred to as the REXSY sequence (Galvosas and Callaghan, 2010), which has both an initial and final CPMG echo train separated by an exchange time, $\tau_{m}$, during which transport can occur between $T_{2}$ domains (Washburn and Callaghan, 2006). 1D $T_{2}$ distributions were measured using the same final CPMG sequence. For the bead packs, the CPMG refocusing pulse spacings $(2 \tau)$ were $70 \mu \mathrm{s}$. By minimizing $\tau$, impacts on the $T_{2}$ relaxation measurements due to diffusion in inhomogeneous magnetic fields, such as susceptibility gradients, are minimized. $T_{2}$ measurements at high magnetic fields ( $7 \mathrm{~T}$ ) are nearly always impacted to some degree by any magnetic susceptibility inhomogeneous fields. Hardware constraints limited $\tau$ to a minimum of $35 \mu \mathrm{s}$. At this short $\tau$ value, 4096 refocusing pulses were used in the final CPMG train to optimally sample signal decay. In the initial CPMG train the number of refocusing pulses varied logarithmically through 128 values ending with 4096 (see Fig. 1). Exchange times, $\tau_{m}$, ranged from $0.1 \mathrm{~ms}$ to $1 \mathrm{~s}$. $T_{1}-T_{2}$ relaxation measurements were acquired by replacing the initial CPMG train with a logarithmically increasing delay time and 180 inversion pulse in order to encode for $T_{1}$ in the 2nd dimension. For the $T_{1}-T_{2}$ and $T_{2}-T_{2}$ experiments on the rock a $\tau$ of $200 \mu$ s was used, a CPMG echo train of 512 points, and an indirect encoding for either $T_{1}$ or $T_{2}$ of 32 points. $T_{2}-T_{2}$ maps were obtained at a range of mixing times between 5 and $250 \mathrm{~ms}$. These parameters were chosen after initial testing showed that using a shorter $\tau$ value and more points led to significant rf heating of the rock. For all the samples, inverse Laplace transformation (ILT) of the data converts the spin relaxation decays into 1D relaxation time distributions or 2D relaxation maps using 2D non-negative least squares (Song et al., 2002; Godefroy and Callaghan, 2003). Finding the optimal values for $n, \tau$ and $\tau_{m}$ is crucial for achieving repeatable and meaningful data sets; depending on the sample the averages were set between 16 and 128 in order to ensure the signal to noise was high enough to ensure reliable ILT.

\section{Results and discussion}

It is important to quantify the growth media's impact on $T_{2}$ measurements before investigating the impact of biofilm growth. How do macromolecules such as proteins, salts and carbohydrates change how water molecules relax inside the porous structure? Will the growth media change the observation of exchange peaks and/or shift the $1 \mathrm{D} T_{2}$ relaxation time distributions? To quantify the BHI growth media's impact on relaxation the SL glass bead pack was used and $T_{2}-T_{2}$ maps were obtained with both deionized water saturation and with the BHI growth media. A set of $T_{2}-T_{2}$ distribution measurements with $\tau_{m}=100 \mathrm{~ms}$ is shown in Fig. 4. The $T_{1}$ values were determined directly using an inversion recovery pulse sequence. The maps in Fig. 4 show that the addition of the BHI growth media impacts the $T_{1}$ and $T_{2}$ of the system and reduces the $T_{1} / T_{2}$ ratio by almost $50 \%$ due to the additional proton exchange sites on the macromolecules (Vaca Chavez et al., 2006). The reduction in $T_{1} / T_{2}$ with the addition of the BHI growth media decreases the intensity of off diagonal peaks for all exchange times (Song et al., 2008).

Fig. 5 shows $1 \mathrm{D} T_{2}$ relaxation distributions for all three bead packs with water, BHI growth media and after biofilm growth. Although the off diagonal peaks disappear with the addition of the $\mathrm{BHI}$ growth media, the $1 \mathrm{D} T_{2}$ relaxation time distributions are not shifted significantly in any of the glass bead packs between the water and BHI growth media. After biofilm growth in the bead pack the exchange peaks did not reappear in the 2D $T_{2}-T_{2}$ maps (not shown), but a significant decrease in the system $T_{2}$ is observed, as indicated in the $1 \mathrm{D}$ distributions of Fig. 5. The distributions are all shifted down by $75-95 \%$ as a result of biofilm growth except in the pure soda lime bead packs which show a smaller reduction.
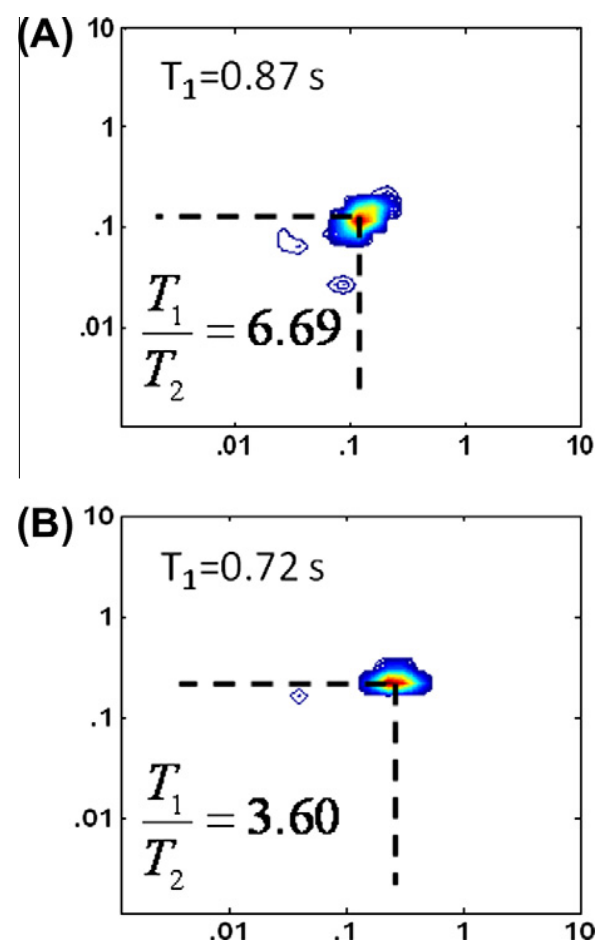

Fig. 4. A set of $T_{2}-T_{2}$ correlation maps ( $\tau_{m}=100 \mathrm{~ms}$ ) are shown for a SL bead pack saturated with water $(\mathrm{A})$ and $\mathrm{BHI}$ growth media $(\mathrm{B})$. 

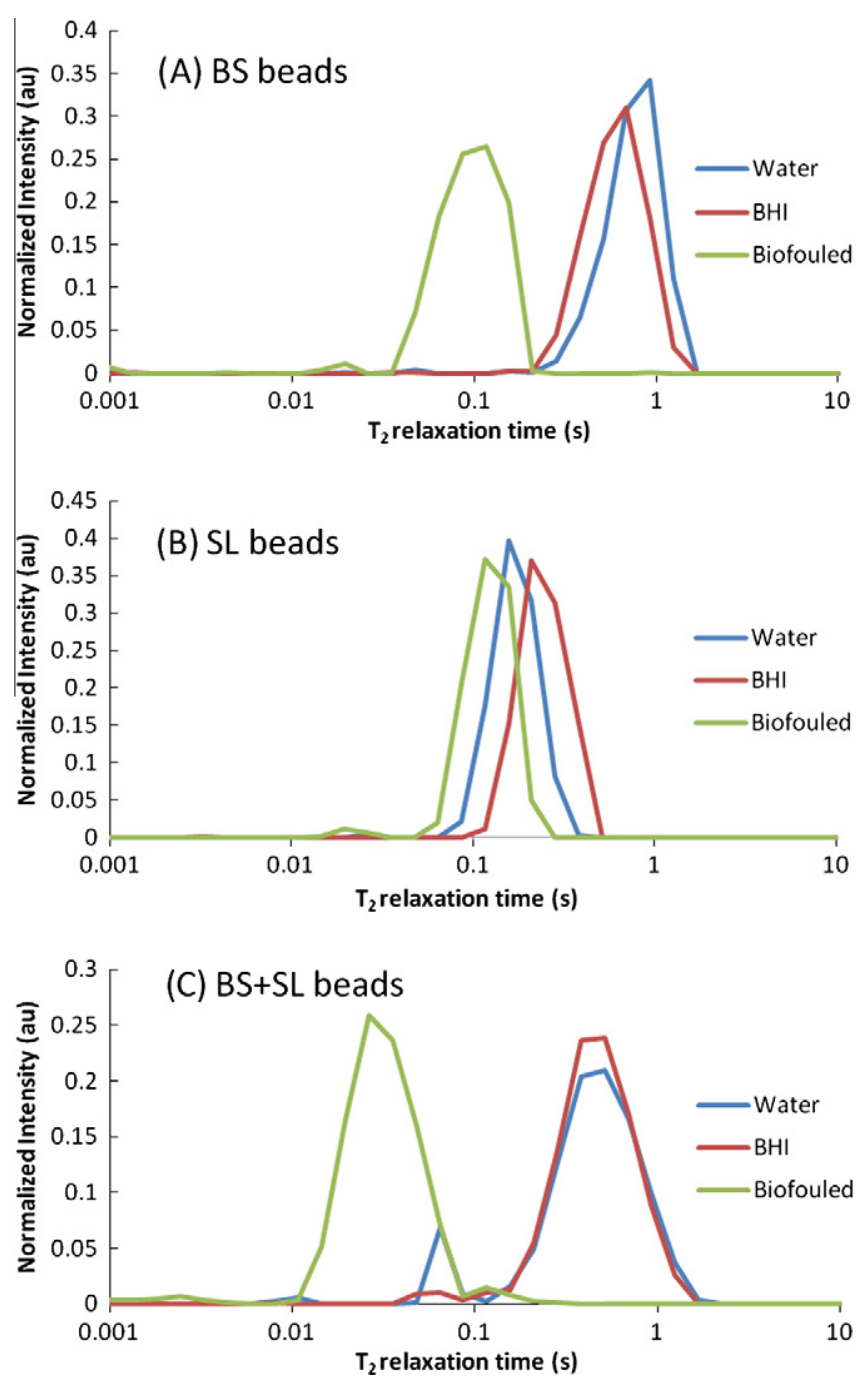

Fig. 5. 1D $T_{2}$ distributions for three model porous systems. The model porous systems were created from $\sim 100 \mu \mathrm{m}$ glass beads (Q.A. Equipment, Ltd., UK). (A) The first bead pack contained borosilicate (BS) glass beads, (B) the second contained soda lime (SL) glass beads, (C) and the third contained a randomised mixture of both types $(\mathrm{BS}+\mathrm{SL})$. 1D $T_{2}$ relaxation distributions for all three bead packs with water and then with BHI growth media and finally after biofilm growth are shown.
$T_{2}-T_{2}$ maps were obtained for a full range of exchange times from $1 \mathrm{~ms}$ to $1 \mathrm{~s}$ for all the bead packs, allowing the water molecules ample time to diffusively sample the entire $100 \mu \mathrm{m}$ pores at longer $\tau_{m}$. Exchange peaks were observed in three bead packs, the water saturated SL and BS + SL and the BHI saturated BS + SL glass bead packs. For these three cases the exchange peaks began to appear after an exchange time of $100 \mathrm{~ms}$. Rather than present all these 2D $T_{2}-T_{2}$ maps, which are similar to those shown in Fig. 4, the magnitude of the exchange peak signal intensity relative to the total signal intensity for the different systems are shown in Fig. 6 as a function of the exchange time.

The time scale associated with the growth of the exchange peaks corresponds to the time taken for water to sample a pore. The $100 \mu \mathrm{m}$ bead pack pore diameters are approximately $80 \mu \mathrm{m}$ based on $a=d_{\mathrm{p}} \Phi /(1-\Phi)$, where the liquid volume fraction $\Phi$ for spherical packing is 0.44 , and $d_{\mathrm{p}}$ is the particle diameter. Assuming unrestricted free diffusion of water, the molecules are expected to diffuse a distance $l_{d}=\sqrt{2 D \tau_{m}}$ between $4 \mu \mathrm{m}$ for a $1 \mathrm{~ms}$ exchange time and $120 \mu \mathrm{m}$ for a $1 \mathrm{~s}$ exchange time. The presence of exchange peaks in the SL and not the BS bead packs are related to the larger $T_{1} / T_{2}$ ratio and stronger susceptibilities associated with the SL beads (Song et al., 2008). It is clear here, as has been previously shown (Mitchell et al., 2007; Washburn and Callaghan, 2006), that if conditions are advantageous for exchange peaks to be visible, then the growth of these exchange peaks as a function of the exchange time will provide access to the average pore size information. However the addition of BHI and the subsequent biofilm growth distort and/or eliminate the presence of exchange peaks and therefore exchange peaks cannot provide information on the structural pore changes that have occurred due to biofilm growth.

The results do however indicate that in these glass bead packs, 1D $T_{2}$ distributions can be used to monitor the extent of biofouling in porous media. The biofilm growth clearly resulted in downshifts in the relaxation time distributions in all the bead packs (Fig. 5) but change in or loss of $T_{2}-T_{2}$ exchange peaks cannot be uniquely attributed to biofouling; however it is clear that it is indicative of a change of pore physical and chemical environment. In certain porous media it may therefore be possible to monitor the 1D $T_{2}$ distribution to determine the percentage of porous media that is biofouled. In a model bead pack, the homogeneous nature of the packing means bacteria can access every pore space and biofilm will grow relatively uniformly throughout media, hence the results seen in Fig. 5 where the presence of a single and reduced $T_{2}$ time

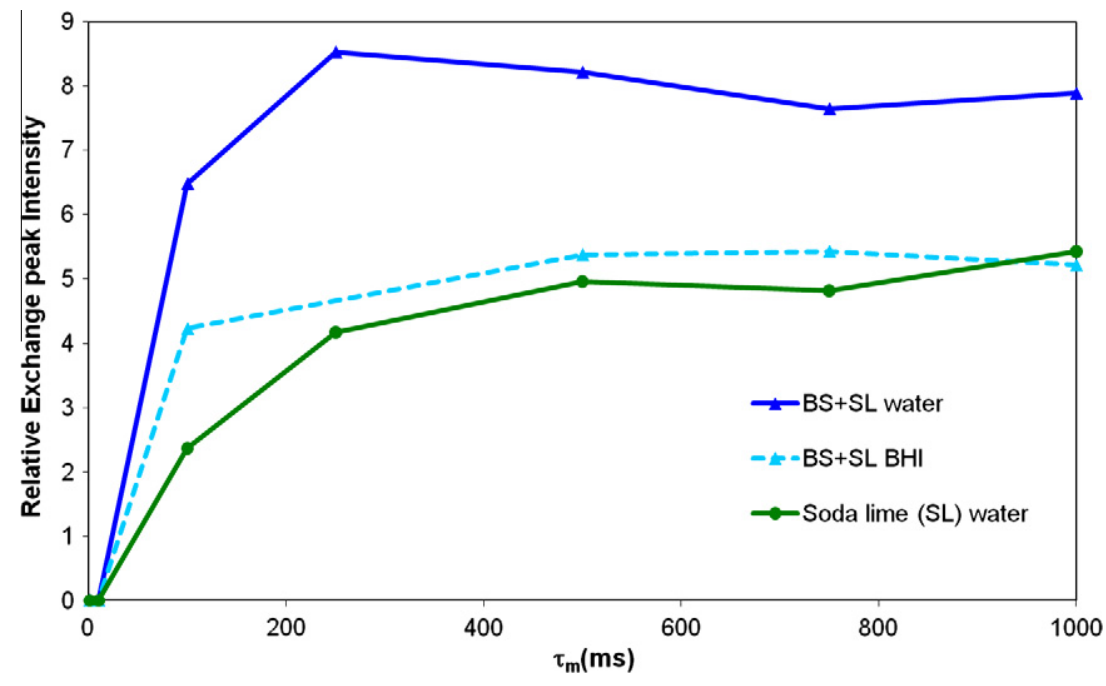

Fig. 6. The magnitude of the exchange peak signals relative to the total signal is shown as a function of the exchange time ( $\left.\tau_{m}\right)$ ranging from $1 \mathrm{~ms}$ to $1 \mathrm{~s}$. 
shows that all the water experiences interaction with biofilm. However, in a natural heterogeneous porous media such as a rock core where the pore spaces are not all equivalently connected, biofilm growth will not necessarily occur in every pore and it can be important to know the percentage of the rock that is biofouled, i.e. the spatial extent of the biofouling. In the homogeneous model bead packs, it is difficult to prevent biofouling from occurring in all the pores. Therefore, in order to demonstrate quantitatively that NMR can determine the extent of biofouling, a simple sample was constructed to ensure the exact same beads and bead packing with only $50 \%$ of the porous media biofouled. A pure BS bead pack was biofouled and then clean BHI saturated BS beads were added upstream of the biofouled column. The NMR rf coil was selectively placed to measure relaxation in the different regions of this

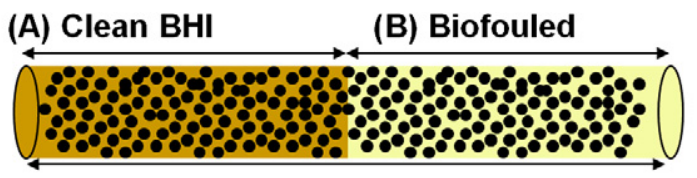

(C) $50 \%$ Biofouled BS

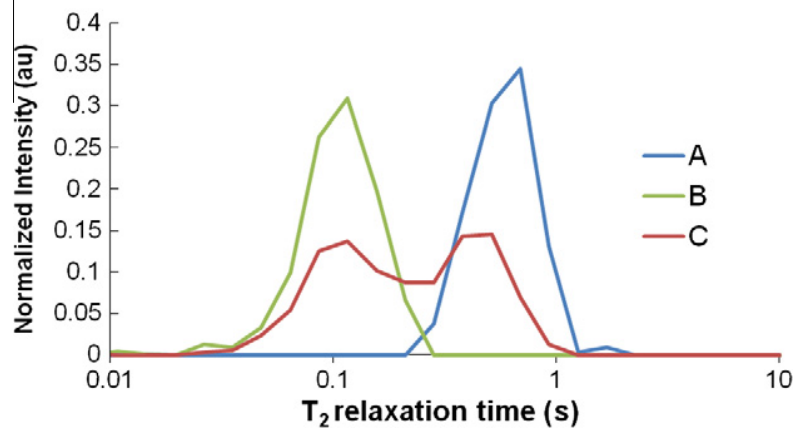

Fig. 7. (top) A schematic indicating the construction of the BS bead pack used to model bead packs with differing extent of biofouling. (A) BHI saturated section, (B) biofouled section, and (C) half BHI saturated and half biofouled section. (bottom) The three relaxation distributions show data from the purely BHI saturated section (A), the half BHI saturated and half biofouled section (B) and the entirely biofouled section (C). controlled heterogeneity sample. Fig. 7 shows how $T_{2}$ relaxation time distribution measurements can be used to measure the extent of biofouling. The three relaxation time distributions shown are from the same $100 \mu \mathrm{m}$ BS glass beads in a clean (i.e. no bacteria) BHI growth media section (A), the boundary section which is half clean $\mathrm{BHI}$ growth media and half biofouled with $B$. mojavensis (C) and the biofouled section (B) (see Fig. 7). The $T_{2}$ values shifted significantly with biofouling, generating an $82 \%$ reduction in $T_{2}$ from the clean BHI saturated section (A) to the biofouled section (B). The distribution of $T_{2}$ times for the $50 \%$ biofouled section (C) is bimodal with two peaks of approximately equal intensity. The interpretation of these data is straightforward due to the homogeneous pore size distribution of the bead packs used in these experiments, but systems with a distribution of pore sizes such as soil or rock would be much more difficult to interpret, as shown in the next section.

To see whether the biological state of the biofilm impacts magnetic relaxation measurements of biomass in a porous media the effect of biofilm starvation and aging was analyzed. The biofouled section of the bead pack was placed back inside the magnet after aging at room temperature for 30 days with no additional growth media. The results (not shown) indicate an increase in $T_{2}$ and narrowing of the $T_{2}$ distribution as the EPS matrix starts to decay. This is in agreement with previous diffusion studies showing an increase in diffusion due to increased molecular mobility as the EPS degrades (Hornemann et al., 2008). The fact that the $T_{2}$ also shifts towards the non-biofouled state is due to a reduction in the availability of rigid proton exchange sites for the water molecules as the biomacromolecules degrade and break down. This also indicates that a downshift in $T_{2}$ times in a glass bead pack can be attributed to intact live biofilm as opposed to decayed biomass.

$T_{2}-T_{2}$ maps were measured for five different Berea rock samples. The range of $T_{2}$ values was consistent in all five rocks tested indicating that the range of pore sizes was similar for all Berea samples and a representative data set is shown in Fig. 8. The development of cross peaks at longer exchange times is consistent with diffusion in internal gradients on the length scale of the pore sizes (de Swiet and Sen, 1994). For example, for a $25 \mathrm{~ms}$ exchange time, water molecules are expected to diffuse about $10 \mu \mathrm{m}$, which is the mean pore size for Berea sandstone (Padhy et al., 2007). Cross
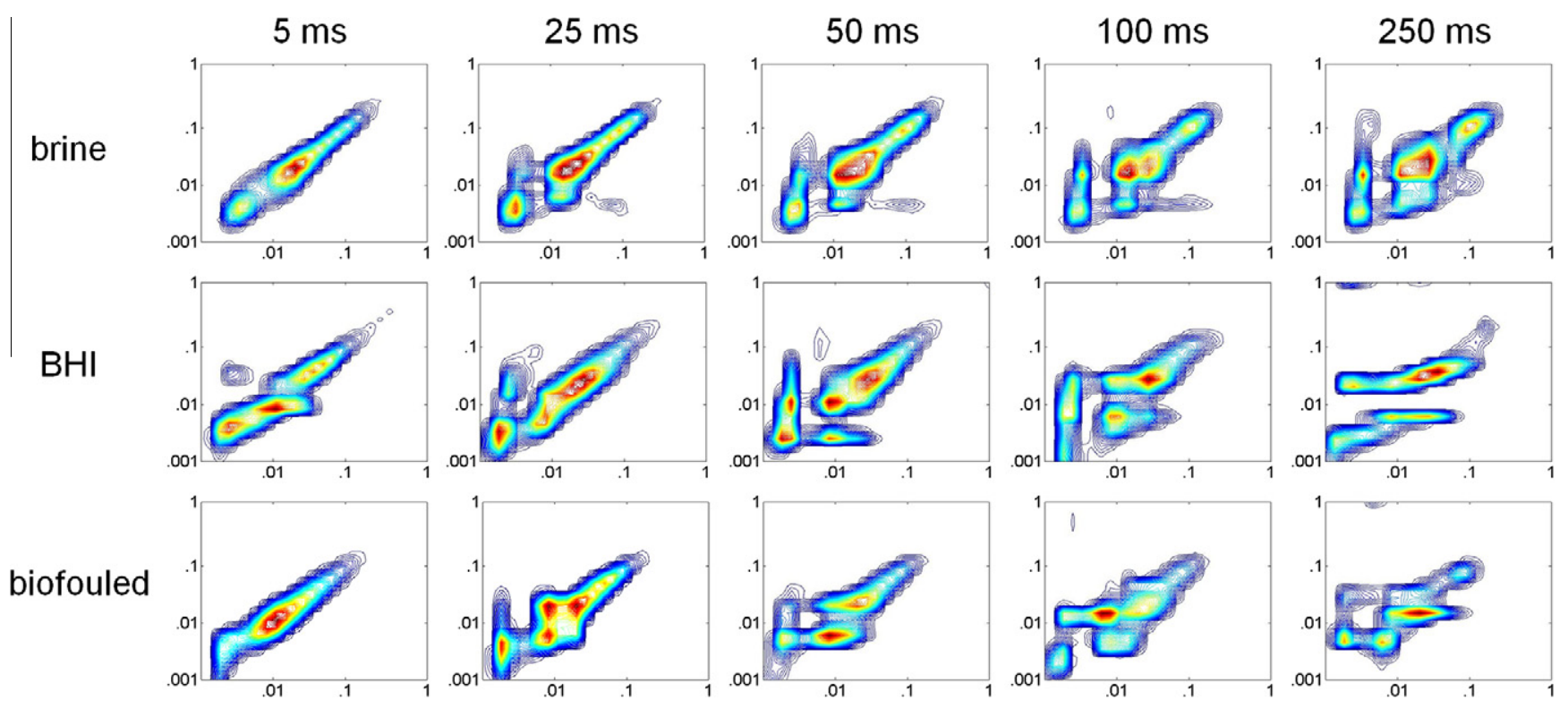

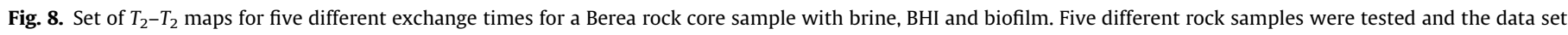
shown is representative of all five samples. 
peaks continue to develop from 50-250 ms, also consistent with the majority of pores ranging from $10-40 \mu \mathrm{m}$ in size. Longer exchange times were not possible because the $T_{1}$ of the molecules in the smaller pores is about $300 \mathrm{~ms}$, and $T_{1}$ relaxation during the exchange time causes signal loss that becomes significant.

However, there were not consistent trends in the changes to the cross peaks with the addition of growth media or biofilm. Statistical analysis of all five samples of Berea rock showed that there were no consistent trends in the distributions that could be attributed to either growth media or biofouling. Any observed changes in any one sample were less than the variations observed between samples. For rocks with pore sizes between 10 and $100 \mu \mathrm{m}$ tested at high field $(7 \mathrm{~T})$, the susceptibility difference between the rock and the fluid will cause internal gradients that will dominate the signal relaxation (Hurlimann, 1998). Since the growth of the biofilm does not significantly alter the diffusion of the water in the pores (Hornemann et al., 2008), the diffusion within the internal gradients will still be the dominant mode of relaxation. Although the measured $T_{2}$ varies over two orders of magnitude consistent with the $T_{2}-T_{2}$ experiments, the $T_{1}$ only varies between $300 \mathrm{~ms}$ and $1 \mathrm{~s}$. This result shows that the internal gradients caused by the high magnetic field affect $T_{2}$ relaxation much more than $T_{1}$ relaxation (Mitchell et al., 2010). The significant impact of magnetic field inhomogeneity complicates direct interpretation of the $T_{2}-T_{2}$ data.

\section{Conclusions}

At high laboratory strength fields ( $\sim 7 \mathrm{~T})$, NMR $1 \mathrm{D} T_{2}$ techniques can be used as a bioassay to confirm the growth of biofilm inside opaque porous media with low susceptibilities such as borosilicate or soda lime glass beads. Additionally we have confirmed that this decrease in $T_{2}$ relaxation time is associated with intact live biofilm as opposed to degraded dead biomass. At these high laboratory strength fields $(\sim 7 \mathrm{~T})$ NMR 2D $T_{2}-T_{2}$ maps cannot be used as a tool to determine the impact on pore sizes and pore connections due to biofilm growth. However in lower susceptibility model bead packs with varying saturating fluid (water, $\mathrm{BHI}$ ) or the presence of biofilm, the growth of exchange peaks in $2 \mathrm{D} T_{2}-T_{2}$ measurements as a function of the exchange time qualitatively indicate sample differences. In natural geological media such as Berea sandstone rock at high magnetic fields, the strong susceptibility gradients dominate the $T_{2}$ distributions and biofilm growth does not systematically change either $1 \mathrm{D} T_{2}$ or $2 \mathrm{D} T_{2}-T_{2}$ distributions and it is not possible to determine the extent of biofouling reliably in this natural geological media using these high field NMR techniques. Future studies will investigate the applicability of $T_{2}$ and $T_{1}$ relaxation measurements to determine the extent of biofouling in natural geological media using a low field NMR system where susceptibility gradients will be significantly reduced.

\section{Acknowledgments}

JDS, SJV and JAH acknowledge support from the US Department of Energy EPSCoR Program under Grant Number DE-FG0208ER46527. SLC acknowledges the support of an NSF Career Award 0642328. The authors thank Paul Callaghan for providing the Laplace inversion software and Robin Gerlach for biofouling equipment and support.

\section{References}

Bayer, J.V., Jaeger, F., Schaumann, G.E., 2010. Proton nuclear magnetic resonance (NMR) relaxometry in soil science applications. The Open Magnetic Resonance Journal 3, 15-26.

Brownstein, K.R., Tarr, C.E., 1979. Importance of classical diffusion in NMR studies of water in biological cells. Physical Review A 19, 2446-2453.

Cunningham, A., Sharp, R.R., Hiebert, R., James, G., 2003. Subsurface biofilm barriers for the contaiment and remediation of contaminated groundwater. Bioremediation 7, 151-164.

de Swiet, T.M., Sen, P.N., 1994. Decay of nuclear magnetization by bounded diffusion in a constant field gradient. Journal of Chemical Physics 100, 55975604.

Fleury, M., Soualem, J., 2009. Quatitative analysis of diffusional pore coupling from $\mathrm{T}_{2}$-store- $\mathrm{T}_{2} \mathrm{NMR}$ experiments. Journal of Colloid and Interface Science 336, 250-259.

Galvosas, P., Callaghan, P.T., 2010. Multi-dimensional inverse Laplace spectroscopy in the NMR of porous media. Comptes Rendus Physique 11,172-180.

Godefroy, S., Callaghan, P.T., 2003. 2D relaxation/diffusion correlations in porous media. Magnetic Resonance Imaging 21, 381.

Godefroy, S., Korb, J.-P., Fleury, M., Bryant, R.G., 2001. Surface nuclear magnetic relaxation and dynamics of water and oil in macroporous media. Physical Review E 64, 021605.

Hornemann, J.A., Lysova, A.A., Codd, S.L., Seymour, J.D., Busse, S.C., Stewart, P.S., Brown, J.R., 2008. Biopolymer and water dynamics in microbial biofilm extracellular polymeric substance. Biomacromolecules 9, 2322-2328.

Hoskins, B.C., Fevang, L., Majors, P.D., Sharma, M.M., Georgiou, G., 1999. Selective imaging of biofilms in porous media by NMR relaxation. Journal of Magnetic Resonance 139, 67-73.

Hurlimann, M.D., 1998. Effective gradients in porous media due to susceptibility differences. Journal of Magnetic Resonance 131, 232-240.

Jaeger, F., Shchegolikhina, A., As, H.V., Schaumann, G.E., 2010. Proton NMR relaxometry as a useful tool to evaluate swelling processes in peat soils. The Open Magnetic Resonance Journal 3, 27-45.

Lee, J.-H., Labadie, C., Springer Jr., C.S., Harbison, G.S., 1993. Two-dimensional Inverse Laplace Transorm NMR: altered relaxation times allow detection of exchange correlation. Journal of American Chemical Society 115, 7761-7764.

Mitchell, J., Griffith, J.D., Collins, J.H.P., Sederman, A.J., Gladden, L.F., Johns, M.L., 2007. Validation of NMR relaxation exchange time measurements in porous media. Journal of Chemical Physics 127, 234701.

Mitchell, A.C., Phillips, A., Hamilton, M., Gerlach, R., Kuszuba, J., Cunningham, A.B., 2008a. Resilience of Bacillus mojavensis planktonic and biofilm communities to supercritical $\mathrm{CO}_{2}$. Journal of Supercritical Fluids 47, 318-325.

Mitchell, A.C., Phillips, A., Hiebert, R., Gerlach, R., Spangler, A.B., Cunningham, A., 2008b. Biofilm enhanced subsurface sequestration of supercritical $\mathrm{CO}_{2}$. International Journal on Greenhouse Gas Control. doi:10.1016/ j.ijggc.2008.05.002.

Mitchell, J., Chandrasekera, T.C., Johns, M.L., Gladden, L.F., Fordham, E.J., 2010 Nuclear magnetic resonance relaxation and diffusion in the presence of internal gradients: the effect of magnetic field strength. Physical Review E 81, 026101.

Monteilhet, L., Korb, J.-P., Mitchell, J., McDonald, P.J., 2006. Observation of exchange of micropore water in cement pastes by two-dimensional $T_{2}-T_{2}$ nuclear magnetic resonance relaxometry. Physical Review E 74, 9061404

Padhy, G.S., Lemaire, C., Amirtharaj, E.S., Ioannidis, M.A., 2007. Pore size distribution in multiscale porous media as revealed by DDIF-NMR, mercury porosimetry and statistical image analysis. Colloids and Surfaces A: Physicochemical and Engineering Aspects 300, 222-234.

Paterson-Beedle, M., Nott, K.P., Macaskie, L.E., Hall, L.D., 2001. Study of biofilm within a packed-bed reactor by three-dimensional magnetic resonance imaging. Methods in Ezymology, 285-305.

Seymour, J.D., Gage, J.P., Codd, S.L., Gerlach, R., 2004. Anomolous fluid transport in porous media induced by biofilm growth. Physical Review Letters 93, 198103.

Sharp, R.R., Stoodley, P., Adgie, M., Gerlach, R., Cunningham, A., 2005. Visualization and characterization of dynamic patterns of flow, growth and activity of biofilms growing in porous media. Water Science and Technology 52, 85-90.

Song, Y.-Q., Venkataramanan, L., Hurlimann, M.D., Flaum, M., Frulla, P., Straley, C., 2002. $T_{1}-T_{2}$ correlation spectra obtained using a fast two-dimensional Laplace inversion. Journal of Magnetic Resonance 154, 261-268.

Song, Y.-Q., Zielinski, L., Ryu, S., 2008. Two-dimensional NMR of diffusion systems. Physical Review Letters 100, 248002.

Vaca Chavez, F., Hellstrand, E., Halle, B., 2006. Hydrogen exchange and hydration dynamics in gelatin gels. Journal of Physical Chemistry B 110, 21551-21559.

von der Schulenburg, D.A.G., Akpa, B.S., Gladden, L.F., Johns, M.L., 2008. Noninvasive mass transfer measurements in complex biofilm-coated structures. Biotechnology and Bioengineering 101, 602-608.

Washburn, K.E., Callaghan, P.T., 2006. Tracking pore to pore exchange using relaxation exchange spectroscopy. Physical Review Letters 97, 175502. 